\title{
Image-guided high-dose-rate intracavitary brachytherapy technique for nasal keloid - a case report
}

\author{
Cheng Huang*, BS, Mingyuan He*, MD, Hongfu Zhao, MS, Prof. Guanghui Cheng, MD, PhD \\ Department of Radiation Oncology. China-Japan Union Hospital of Jilin University, Changchun, China \\ *These two authors contributed equally to this work.
}

\begin{abstract}
The aim of this study is to report an uncommon case of nasal keloid treated with adaptive image-guided highdose-rate (HDR) intracavitary brachytherapy. A 34-year-old female presented with nasal keloid after facial trauma was treated with scar resection and HDR intracavitary brachytherapy with a customized eccentric applicator. The total dose was 21 Gy in 6 fractions. During 3 years of follow-up, there was no evidence of recurrence at the local site, and no complications. In conclusion, HDR intracavitary brachytherapy is an effective way to prevent the recurrence of nasal keloids post-surgery.

J Contemp Brachytherapy 2018; 10, 3: 279-282 DOI: https://doi.org/10.5114/jcb.2018.76838
\end{abstract}

Key words: brachytherapy, customized applicator, nasal keloid.

\section{Purpose}

Keloids arise from excessive fibroblast proliferation after trauma or a surgical incision [1], which extend beyond the borders of the original wound, usually causing ingling, itching, and cosmetic disfigurement. Keloids often occurred in the chest, shoulders, upper back, head and neck areas (especially ear lobes), and limbs; furthermore, keloids located in the nasal mucosa are a rare case. The treatment of keloids has always been a challenge in plastic surgery, because of high recurrences rate of $45-100 \%$ after excision alone [2]. The recurrence of keloids may be caused by the upregulation of fibrotic markers of keloids and surrounding normal skin fibroblasts that increase collagen and fibronectin deposition [3]. Post-operative adjuvant therapy is the main treatment strategy for keloids, including radiotherapy, corticosteroid injections, silicone occlusive dressings, laser therapy, pressure therapy and cryotherapy [4], significantly reduces the recurrence rate. Radiotherapy is considered one of the most effective adjuvant therapy $[5,6]$. The mechanism of action is that radiation induces fibroblasts damage, resulting in the reduction of collagen fibers and matrix synthesis. Radiotherapy can be delivered with superficial kilovolt X-rays, electron beams, or brachytherapy. In China, the external beam radiotherapy is widely used in clinical application for keloids, compared with the brachytherapy [7]. In this context, we propose a technique using HDR intracavitary brachytherapy to treat nasal keloids after surgery.

\section{Case report}

\section{Patient presentation}

The case of a 34-year-old female with nasal keloid after surgery is presented. The patient came to the otolaryngologic department after facial trauma, and received debridement and suturing. One year later, she suffered from nasal obstruction and was referred to our clinic. Computed tomography (CT) scan showed a keloid located in the inferior nasal concha (Figure 1). She underwent nasal deformities correction and scar resection. Two days later, she received an adaptive image-guided high-doserate (HDR) intracavitary brachytherapy.

\section{Treatment modalities}

The customized eccentric applicator consisted of solidified alginate and a flexible tube (Figure 2A). The applicator was inserted into left nasal cavity referencing the preoperative CT-images and the scar, ensured the tube was closed to the scar but away from the nasal septum (Figure 2B). Fine-pitch $(2 \mathrm{~mm}) \mathrm{CT}$ images were acquired and transferred to the treatment planning computer. Clinical target volume (CTV) consisted of the surgical wound plus $5 \mathrm{~mm}$ margin, retracting the side of cavity, and the nasal septum was the organs at risk (OAR). Then, a CT-based treatment plan was created and optimized on the treatment planning system Oncentra v. 4.3 (Nucletron, Veenendaal, The Netherlands). The prescribed dose was $21 \mathrm{~Gy}$ in 6 frac-
Address for correspondence: Prof. Guanghui Cheng, MD, PhD, China-Japan Union Hospital of Jilin University, No.126, Xian-Tai Street, Changchun 130000, China, phone: +86 13610712080,

e-mail: chengguanghuifl@163.com
Received: 26.02 .2018

Accepted: 04.06 .2018

Published: 30.06 .2018 

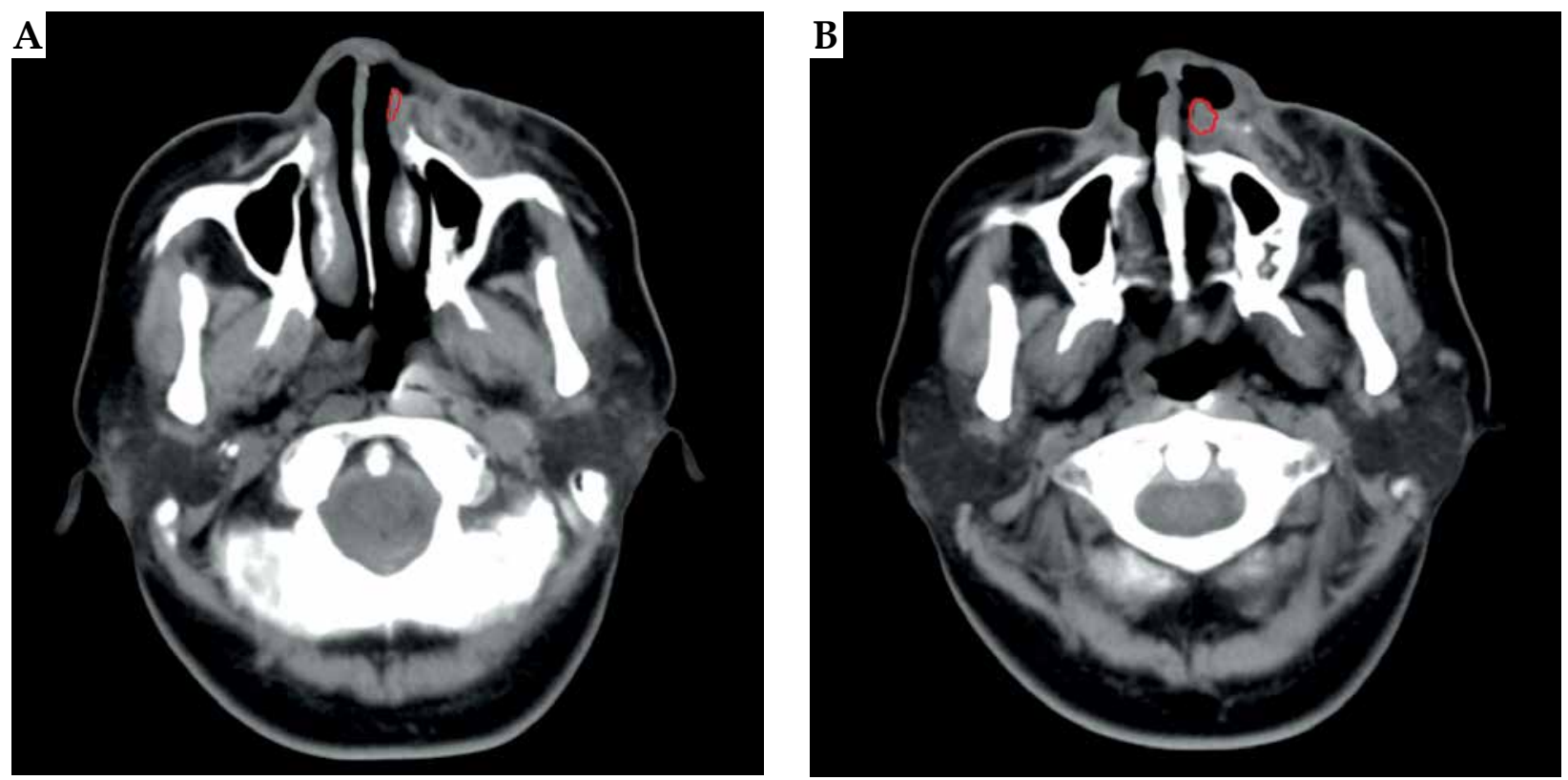

Fig. 1. Preoperative computed tomography showed the lesion located in the left inferior nasal concha

tions during 3 consecutive days (3.5 Gy each fraction, twice a day with 6 hours interval). The applicator was in the same position for the whole treatment time, which was well tolerated and fixed tightly. Before every fraction, we inserted a CT marker system set (Elekta company, Stockholm, Sweden) into the catheter and followed this with a CT simulation to check the exact position of the catheter. Dose volume histogram $(\mathrm{DVH})$ parameters were used for evaluating the target volume and OAR. The dose distribution to target volume and OAR are presented in Figure 3. The biologically effective doses (BED) was calculated using the linear quadratic equation, with $\alpha / \beta=10$ [8].

\section{Results}

The DVH showed that $\mathrm{V}_{100}$ and $\mathrm{V}_{150}$ (volume ratio of the target receiving $100 \%$ or $150 \%$ of the therapeutic dose) of CTV was $90.3 \%$ and $42.7 \%$, respectively. The $\mathrm{D}_{90}$ (mini- mum dose delivered to $90 \%$ of target volume) of CTV was $3.51 \mathrm{~Gy}$. The $\mathrm{D}_{0.1 \mathrm{cc}}$ (minimum dose delivered to $0.1 \mathrm{~cm}^{3}$ of target volume) and the $\mathrm{D}_{\text {mean }}$ of nasal septum was $3.50 \mathrm{~Gy}$ and $2.45 \mathrm{~Gy}$, respectively. Brachytherapy was well tolerated during the treatment, and the patient presented only a mucosal erythema and pruritus (RTOG/CTCAEs grade I acute mucosal response) one week after the treatment. In the first 3 months, the patient suffered nasal xerosis (RTOG/CTCAEs grade I acute mucosal response), which was resolved without any treatment. At 3 years of follow-up, there was no sign of local recurrence, and no late complication was reported.

\section{Discussion}

Currently, the external beam radiotherapy is widely used for post-operative treatment of keloids, but some studies favor brachytherapy rather than superficial
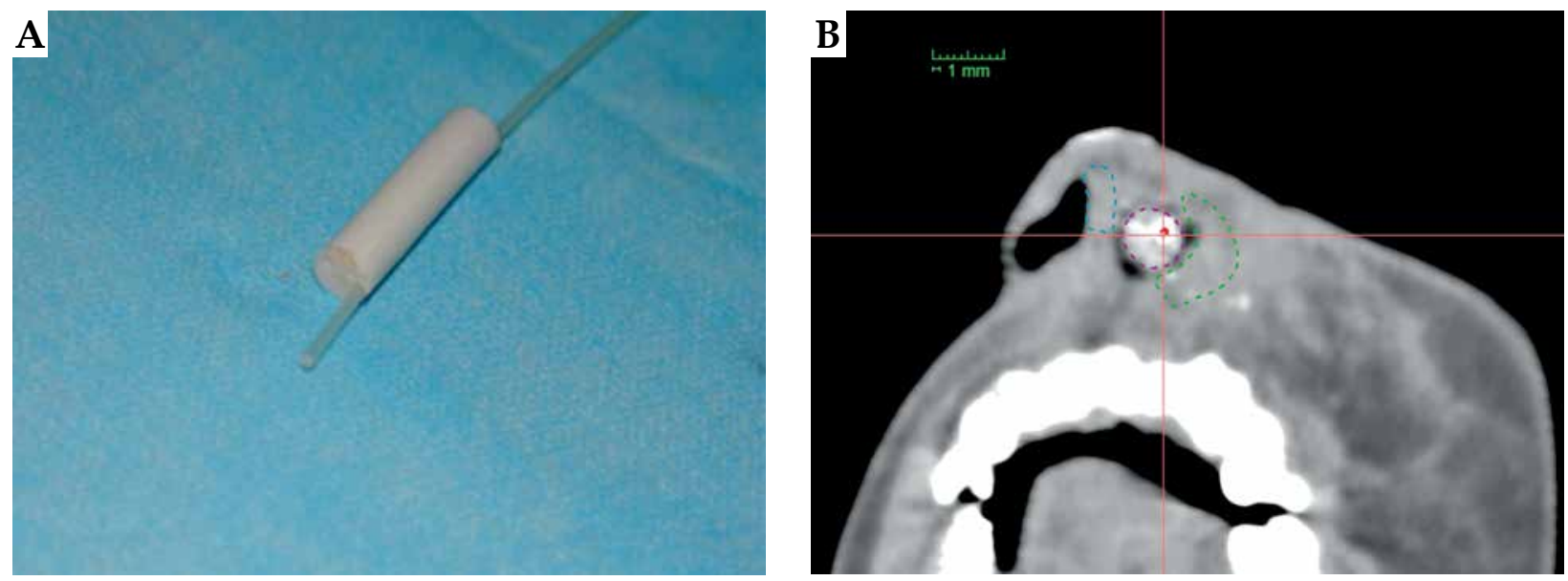

Fig. 2. A) The customized eccentric applicator. B) The tube was closed to the surgery margin but kept away from the nasal septum 

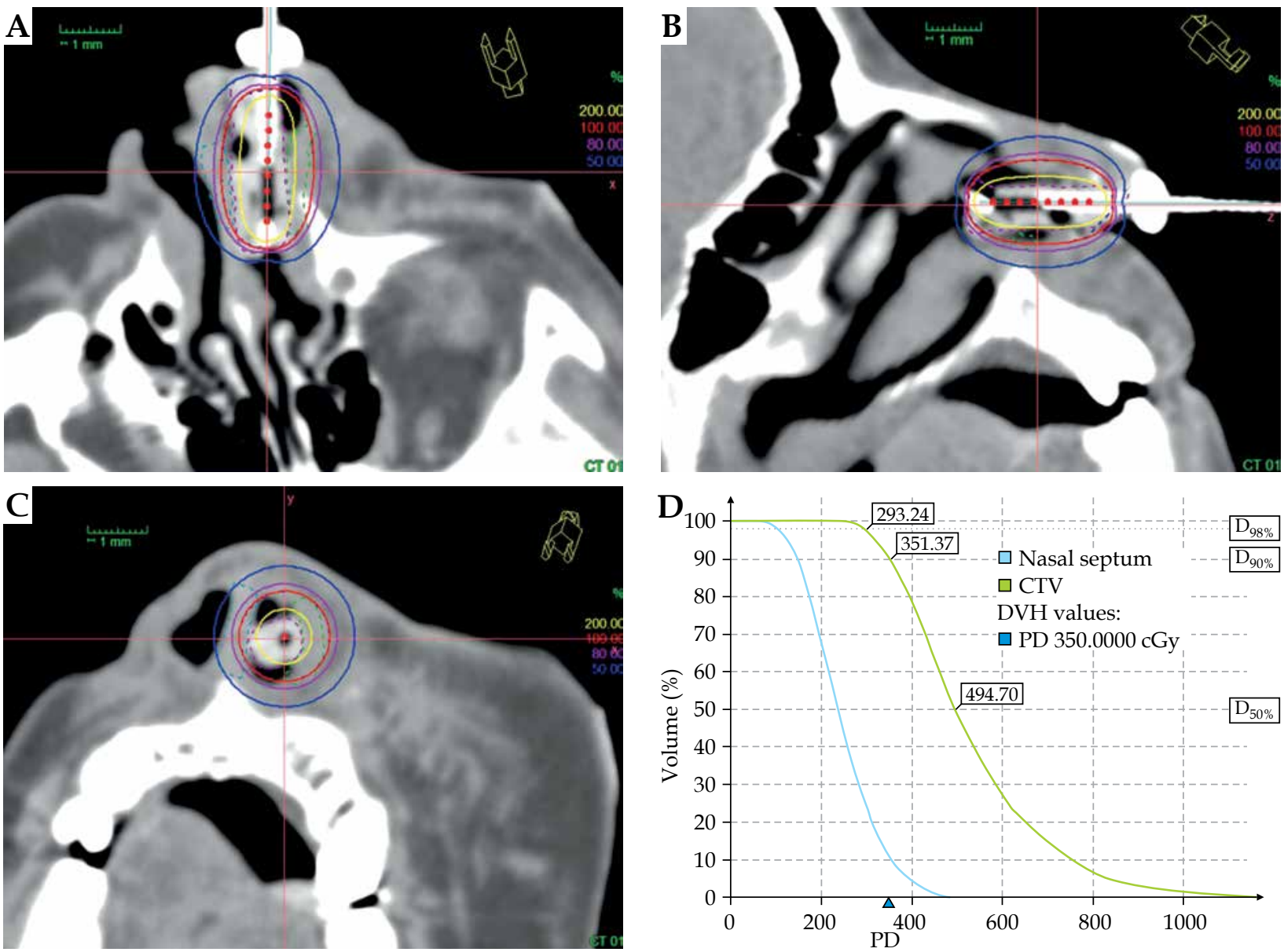

Fig. 3. A-C) The target volume dose distribution of horizontal, sagittal, and coronal positions. D) The dose volume histogram parameters

X-rays or electron beams [6,9]. A meta-analysis systematic review [10] indicated that brachytherapy had a lower recurrence rate $(15 \%)$ when compared to electron beam and X-ray therapy (23\% and $23 \%$, respectively) for keloids. Guix et al. [9] reported 169 cases of keloids treated by interstitial brachytherapy, with a low recurrence rate of $4.7 \%$ and satisfactory cosmetic results. Vivante et al. [11] reported a case with six lesions on the ear (two keloids and four hypertrophic scars) treated with a ${ }^{32} \mathrm{P}$-patch brachy-radiotherapy, reaching satisfactory clinical result and no complications. Clearly, brachytherapy performed by interstitial and superficial technique is an effective and safety adjuvant therapy for keloids after surgery, but intracavitary brachytherapy has not been previously reported.

There is no standard recommended schedule for dosage and fractionation. Kal and Veen [8] reviewed the literature and converted different fractionated scheme into BED by LQ concept $(\alpha / \beta=10)$, and suggested that a BED value of at least $30 \mathrm{~Gy}$ was required for keloids. Flickinger [5] retrospectively analyzed 2,515 cases of keloids treated by external beam radiotherapy after surgery and calculated a low $\alpha / \beta$ ratio of 2.08 Gy for keloids, which was similar to late reacting tissues. This suggested that the best way to deliver post-operative radiation is to give fewer fractions and higher doses per fraction. Misbah et al. [12] divided 65 cases of keloids into 4 groups, in the scheme of $1 \times 8$ Gy (7 cases), 1 × 10 Gy (19 cases), 3 × 5 Gy (20 cases), $3 \times 6$ Gy (19 cases), and found that the recurrence rates were $42.8 \%, 10.5 \%, 15 \%$, and $10.5 \%$, respectively. For the choice of dose rate, Cicco et al. [13] reported 50 keloids cases treated with HDR brachytherapy with a median dose of $12 \mathrm{~Gy}$, while 46 keloids treated with low-dose-rate (LDR) brachytherapy in a median dose of 16 Gy resulted in a similar recurrence rate, but HDR treatment had a better symptomatic relief. Moreover, the treatment time of HDR was short, which was convenient for clinical application.

In this case, the keloid was located in the nasal cavity, which was a challenge because of its special anatomical position. If X-ray irradiation was applied, not only would the cavity effect influence the dose distribution, but also it would lead high-dose to the surrounding normal tissues. With electron beam given the scar, which had a deep location, this would result in a high skin dose. Interstitial brachytherapy using catheters inserted at the time of surgery is commonly used for post-operative treatment of keloids [14], but the keloid in our case was located in a special anatomical site that was difficult to treat with interstitial method. Intracavitary brachytherapy is com- 
monly used in the treatment of human lumen structural organ tumors, and provides a high-dose radiation to the target but a lower dose to normal tissue. We proposed an HDR intracavitary brachytherapy for nasal keloid treatment. A customized eccentric applicator was created to reduce the dosage to nasal septum. The DVH showed that CTV was covered with high-dose irradiation, while the dosage of nasal septum was relatively low. Considering that fractionated high-dose will increase the side effect of mucosa as an acute reacting tissue, we used a scheme of $6 \times 3.5$ Gy and BED was up to 28.35 Gy for $\alpha / \beta=10$. The patient came to our department after 48 hours because of personal reasons. Most of the literatures recommend that post-operative radiotherapy should start as soon as possible, and the first session should be on the same day as surgery [14], there are also some studies that have not found a correlation between the interval time and excision, radiotherapy and therapeutic outcome $[15,16]$. Treatment was well tolerated and at 3 years of follow-up, there was no local recurrence or late side effects.

\section{Conclusions}

Brachytherapy, using adaptive applicator, is an effective and well tolerated treatment for delivering postoperative radiotherapy for nasal cavity keloids. This method can be considered for similar challenging clinical scenarios.

\section{Acknowledgements}

This study was partially supported by the National Natural Science Foundation of China (31600679), Project of Science and Technology Department of Jilin Province (20090458), Project of Health and Family Planning Commission of Jilin Province ( 2014ZC054), Bethune Special Research of Science and Technology Department of Jilin Province (20160101079JC), and Horizontal Project of Jilin University $(2015373,2016220101000686)$.

\section{Disclosure}

The authors report no conflict of interest.

\section{References}

1. Garg MK, Weiss P, Sharma AK et al. Adjuvant high dose rate brachytherapy (Ir-192) in the management of keloids which have recurred after surgical excision and external radiation. Radiother Oncol 2004; 73: 233-236.

2. Jie S, Xin L, Sun Y et al. Hypofractionated electron-beam radiation therapy for keloids: retrospective study of 568 cases with 834 lesions. J Radiat Res 2015; 56: 811-817.

3. Ashcroft KJ, Syed F, Bayat A. Site-specific keloid fibroblasts alter the behavior of normal skin and normal scar fibroblasts through paracrine signaling. PLoS One 2013; 8: e75600.

4. Nast A, Eming S, Fluhr J et al. German S2k guidelines for the therapy of pathological scars (hypertrophic scars and keloids). J Dtsch Dermatol Ges 2012; 10: 747-762.

5. Flickinger JC. A radiobiological analysis of multicenter data for postoperative keloid radiotherapy. Int J Radiat Oncol Biol Phys 2011; 79: 1164-1170.

6. Ogawa R. The most current algorithms for the treatment and prevention of hypertrophic scars and keloids. Plast Reconstr Surg 2010; 125: 557-568.
7. Shen J, Lian X, Sun Y et al. Hypofractionated electron-beam radiation therapy for keloids: retrospective study of 568 cases with 834 lesions. J Radiat Res 2015; 56: 811-817.

8. Kal HB, Veen RE. Biologically effective doses of postoperative radiotherapy in the prevention of keloids. Dose-effect relationship. Strahlenther Onkol 2005; 181: 717-723.

9. Guix B, Henríquez I, Andrés A et al. Treatment of keloids by high-dose-rate brachytherapy: A seven-year study. Int J Radiat Oncol Biol Phys 2001; 50: 167-172.

10. Mankowski P, Kanevsky J, Tomlinson J et al. Optimizing radiotherapy for keloids: a meta-analysis systematic review comparing recurrence rates between different radiation modalities. Ann Plast Surg 2017; 78: 403-411.

11. Vivante H, Salgueiro MJ, Ughetti R et al. 32P-patch contact brachyradiotherapy in the management of recalcitrant keloids and hypertrophic scars. Indian J Dermatol Venereol Leprol 2007; 73: 336-339.

12. Ahmad M, Ahmad H, Khattak MR et al. Postoperative single versus multiple fractions high-dose rate iridium-192 surface mould brachytherapy for keloid treatment: a comparative study. J Radiother Pract 2018; 17: 60-65.

13. De Cicco L, Vischioni B, Vavassori A et al. Postoperative management of keloids: Low-dose-rate and high-dose-rate brachytherapy. Brachytherapy 2014; 13: 508-513.

14. Guinot JL, Rembielak A, Perez-Calatayud J et al. GEC-ESTRO ACROP recommendations in skin brachytherapy. Radiother Oncol 2018; 126: 377-385.

15. Sakamoto T, Oya N, Shibuya K et al. Dose-response relationship and dose optimization in radiotherapy of postoperative keloids. Radiother Oncol 2009; 91: 271-276.

16. Viani GA, Stefano EJ, Afonso SL et al. Postoperative strontium-90 brachytherapy in the prevention of keloids: results and prognostic factors. Int J Radiat Oncol Biol Phys 2009; 73: 1510-1516. 